\title{
TO COMPARE THE PREVALENCE OF PERSONALITY DISORDERS IN A COMMUNITY FROM RURAL AND URBAN AREAS: A CROSS-SECTIONAL STUDY IN SOUTH KARNATAKA, INDIA
}

\author{
Bhuvana C. Revappala ${ }^{1}$
}

${ }^{1}$ Consultant Psychiatrist, Department of Psychiatry, Wellspring Mind Care, Sharavathinagara, Shimoga.

\begin{tabular}{l} 
ABSTRACT \\
\hline BACKGROUND \\
There is a lack of study on the prevalence of personality disorders from rural areas since the degree of willingness of people \\
from rural areas to use mental health services, self-report of mental illness and availability of these services in these areas is very \\
less. These personality disorders frequently co-occurs with other psychiatric disorders, which can complicate their treatment and \\
worsen their prognosis.
\end{tabular}

AIMS

To compare the prevalence of personality disorder among rural and urban community in South Karnataka and to estimate is there any differences in quantities and qualities of personality disorders among two groups.

\section{MATERIALS AND METHODS}

An equal number of 300 subjects from urban and 300 subjects from rural areas aged 18-75 years were screened by using SAPAS to identify the cases and further diagnosis was confirmed by using DSM-V TR2 in a two-phase survey. A Chi-square test is used for statistics.

\section{RESULTS}

The weighted prevalence of overall personality disorder in urban areas was $19.4 \%$ and $17.2 \%$ in rural areas, but not significant statistically. But statistical significance was found for cluster subgroup, where cluster-C subgroup of personality disorders were found to be highest prevalent among rural areas and cluster-B was found to be highest among urban.

\section{CONCLUSIONS}

Since the rural mental health problems tend to be less visible than urban and can lead to the erroneous conclusion that rural areas have a lesser need for mental health services. Our study has found that there is the almost equal prevalence of personality disorder in rural and urban areas, but differences were in (quality) cluster subgroups of personality disorders. The reasons for these different findings need to be studied further in detail.

\section{KEYWORDS}

Personality Disorders, Cluster Subtypes, Rural and Urban Areas.

HOW TO CITE THIS ARTICLE: Revappala BC. To compare the prevalence of personality disorders in a community from rural and urban areas: a cross-sectional study in South Karnataka, India. J. Evolution Med. Dent. Sci. 2016;5(81):6046-6051, D0I: $10.14260 /$ jemds/2016/1365

\section{BACKGROUND}

Knowledge of personality disorder is important for all medical practitioners, because it affects a significant minority of individuals and may influence overall clinical management whether in psychiatric or primary care settings. PD has been associated with a number of adverse consequences in the general population including marital problems, high rates of separation, divorce, occupational difficulties, criminality, poor quality of life for the individual and his/her family and increased risk of mortality through suicide, homicide and accidents. ${ }^{1,2} \mathrm{~A}$ personality disorder is defined as "pervasive, inflexible and maladaptive" collections of traits that interfere

Financial or Other, Competing Interest: None.

Submission 15-09-2016, Peer Review 28-09-2016,

Acceptance 01-10-2016, Published 08-10-2016.

Corresponding Author:

Dr. Bhuvana C. Revappala,

Wellspring Mind Care,

Sharavathinagara,

Shimoga.

E-mail: doc.barbie@yahoo.in

DOI: $10.14260 /$ jemds $/ 2016 / 1365$ with an individual's ability to function productively and that of behaviour deviates markedly from the expectations of the individual's culture. Personality disorders are usually conceived as personality systems or structures that are not well adapted to social requirements or function poorly in their specific environment (Magnavita, 2004). These difficulties are supposed to have an impact on several life domains (Cognition, emotion and impulse regulation and interpersonal functioning) to be stable over time to induce a mental pain or have a negative impact on a person's wellbeing and to have started during adolescence or early adulthood. However, few individuals with a personality disorder make contact with psychiatric services compared to other psychiatric conditions and their probability of withdrawing from treatment is considerably higher. Moreover, when a personality disorder is present the treatment of other coexisting psychiatric or medical conditions is frequently more complicated, lengthier or less successful; a pattern that may at times be due to lack of recognition of the personality disorder. ${ }^{3}$ The community based epidemiological studies of psychiatric disorders provide important information about the public health burden of these problems. 
Although, the frequency of treatment seeking for psychiatric disorders may be increasing, epidemiological studies have indicated that most patients in the community do not get treatment for psychiatric disorders. Differences between the general population and clinical population, epidemiological studies might be greatest when we examine the prevalence of disorders and diagnostic co-morbidity because help seeking is related to co-morbidity. ${ }^{4}$

In addressing the above mental health issues, it is important to revisit the definitions of "rural" and "urban" areas at least briefly, because they can be relatively complex and cut across a variety of social, cultural and economic issues. "Urban" generally denotes cities and implies cities of any size with a total population of at least 100,000. "Rural" areas are perhaps even more difficult to define and are more characterised than urban areas, being outside of a city or town greater than 2,500 persons.

A lack of anonymity in rural communities and the perceived social stigma associated with mental illness may prevent the seeking of treatment, regardless of referred by the general physician. This stigma associated with seeking mental health treatment is frequently identified as a most serious barrier to care for rural residents than in urban ones.

Several studies have suggested that the prevalence of Mental Health Problem (MBH) may be greater in urban areas (Paykel, Abbott, Jenkins, Brugha and Melzer, 2000; Sundquist, Frank and Sundquist, 2004; Peen et al, 2007), whereas several others (Center on An Aging Society, 2003) suggest these problems may be more prevalent in rural areas. Furthermore, a few studies have suggested that the overall prevalence of MBH problems may not differ between urban and rural areas, but rather the types of $\mathrm{MBH}$ problems may vary (Peen et al, 2006).4,5

The prevalence of Personality Disorders (PDs) is fairly high even in the general population, where it ranges from 3 to $15 \%$. In psychiatric populations its prevalence is about $30 \%$ among outpatients, $40 \%$ among inpatients and up to $70 \%$ in prison psychiatric populations. Most studies assessing prevalence rates of PD have focused on clinical populations. A study by Lenzenweger et al has presented the first highquality estimate of PD in a non-clinical population (11\%). Since then a number of studies have attempted to determine prevalence rates in community samples. In the United States, community prevalence rates ranging from $9 \%$ to $15.7 \%$ have been found. Similar rates of $10.0 \%$ in Germany, $11.2 \%$ in Sweden and $13.4 \%$ in Norway have been documented. ${ }^{6}$

Since most of the above studies have done only in urban areas, whereas prevalence studies of personality disorders in rural areas were nil or negligible. We are therefore comparing the prevalence of personality disorder among rural and urban population including demographic characteristics, use of clinical and institutional services, which has divided into three cluster subgroups of personality disorders based on the DSM-V classification systems in a two-phase survey at Shimoga city and 3 villages of Shimoga district.

\section{METHODS}

\section{Source of Sample}

The present study is a community-based cross-sectional study, in which the city of Shimoga was taken as an urban area and three villages namely Mandgadde, Rippanpete and
Ayanur near the city of Shimoga were considered as rural areas.

The sample has been selected randomly from the people who visited urban health centres in Shimoga and rural health centres from three villages of Shimoga district, namely Mandgadde, Rippanpete and Ayanur by taking permission from higher authority of respective health centres.

\section{SAMPLING PROCEDURE}

The interview has been conducted by the Psychiatrist. All the patients including their family members who visited health centres were screened initially by using SAPAS scale to assess the personality disorders. Those who met the SAPAS scale criteria for PD had been re-interviewed further by using DSM$\mathrm{V}$ diagnostic criteria to confirm the diagnosis. This is a twostage procedure to confirm the diagnosis. We avoided the door-door surveys in residential areas, since many of the family members usually not residing at home during daytime for survey which leads to difficult to collect samples and also it takes too long time, whereas in health centres we can get at-least 2-3 family members for personality assessment of individuals where we can get mutual informants for each individuals of family members. The present study was conducted in the selected Shimoga city of Karnataka state as urban respondents. For the rural sample three villages namely Mandgadde, Rippanpete and Ayanur.

\section{Sample Size}

Out of the 600 total numbers of samples, 300 were selected from the city of Shimoga as urban respondents and remaining 300 samples were collected from rural areas, 100 from each of three villages namely Mandgadde, Rippanpete and Ayanur. Since we could not collect the entire 300 sample from a single village, because many refused to participate in an interview. Hence, we selected three villages to make the equal number of samples in both urban and rural areas in order to avoid bias.

\section{Age Group}

18-75 years of either sex. Informed consent was obtained from all the patients who were included in the study. Other socio-demographic details like education level, occupation, income and marital status of all the patients visiting to the health centres has been collected.

\section{Statistical Method}

The Chi-square test was used. A p-value of $<0.05$ was considered as statistically significant.

Tools: 1 . SAPAS, 2. DSM-V.

SAPAS: The Structured Assessment of Personality Abbreviated Scale.7,8,9

The SAPAS is a better scale to be used in epidemiological research as part of a two-stage procedure for case identification. SAPAS has a Sensitivity of $87.5 \%$; Specificity of $85.7 \%$. It is an eight-item screening interview for assessment of personality. Each item is worded as a question to be answered with yes or no (e.g. item 1: "In general, do you have difficulty making and keeping friends?").

When the response is given that indicates pathology (i.e. yes to item 1), the interviewer must follow up by asking if 
that is true in general. The SAPAS will normally be completed in less than a minute.

As the SAPAS is a set of indicators covering multiple areas, it is not designed to be one-dimensional. Rather, the SAPAS is designed to cover different areas of personality. The SAP allows an ICD-10 or DSM-V diagnosis of personality disorder to be made (World Health Organisation, 1992; American Psychiatric Association, 1994). The screen itself should not be used to make a diagnosis of personality disorder or cluster of personality disorders and we have to re-interview the patients whose scoring was more than 3 by using DSM-V to make a complete diagnosis of personality disorders as a twophase procedure. DSM-VTR-Diagnostic and statistical manual of mental disorders, $5^{\text {th }}$ edition. ${ }^{10}$

The diagnosis has been confirmed further by using DSM-V criteria, where there are three cluster subgroups. Cluster- $A$ is characterised by odd and eccentric behaviours and includes paranoid, schizoid and Schizotypal PDs. Cluster-B is characterised by dramatic and erratic emotions and behaviours and includes histrionic, narcissistic, antisocial and borderline PDs. Finally, Cluster-C is characterised by anxious and fearful feelings and attitudes and includes avoidant, dependent and obsessive-compulsive PDs.

\section{Inclusion Criteria}

- All the age group above 18 years and below 75 years.

- Those who have substance abuse like alcohol, tobacco and other substance abuse.

- Both sexes.

- Married and unmarried.

\section{Exclusion Criteria}

The individuals who had severe medical or mental illness, too old aged people, brain injury, intracranial disorders like brain tumours which results in organic personality changes, etc., which interfered with communication were excluded from the study.

\section{RESULTS AND DISCUSSION}

To our knowledge, the present study seems to be the first to report specifically on the prevalence of personality disorders between urban and rural areas in South India, where these studies were negligible or nil in India. A very few studies (Rossier J, et al 2013; Paris' 1998) have been done on personality and personality disorder to describe the relationship between personality disorder and culture among the rural and urban areas and has been concluded that prevalence rate of PDs are supposed to be culturally dependent and was hypothesised that PDs of cluster $\mathrm{C}$ should be more frequent in the rural sample and PDs of cluster $B$ should be more frequent in the urban sample (Paris; 1998) rather than estimating prevalent rates. ${ }^{11,12}$

\begin{tabular}{|c|c|c|}
\hline & Rural & Urban \\
\hline Cluster A & $0.3 \%$ & $1.9 \%$ \\
\hline Cluster B & $1.1 \%$ & $17.3 \%$ \\
\hline Cluster C & $15.9 \%$ & $0.2 \%$ \\
\hline Total & $\mathbf{1 7 . 3 \%}$ & $\mathbf{1 9 . 4 \%}$ \\
\hline Significant Statistically & $\mathrm{p}<0.05$ & \\
\hline
\end{tabular}

\begin{tabular}{|c|c|c|}
\hline & Rural & Urban \\
\hline Females & $69.2 \%$ & $66.1 \%$ \\
\hline Males & $30.8 \%$ & $34.9 \%$ \\
\hline
\end{tabular}

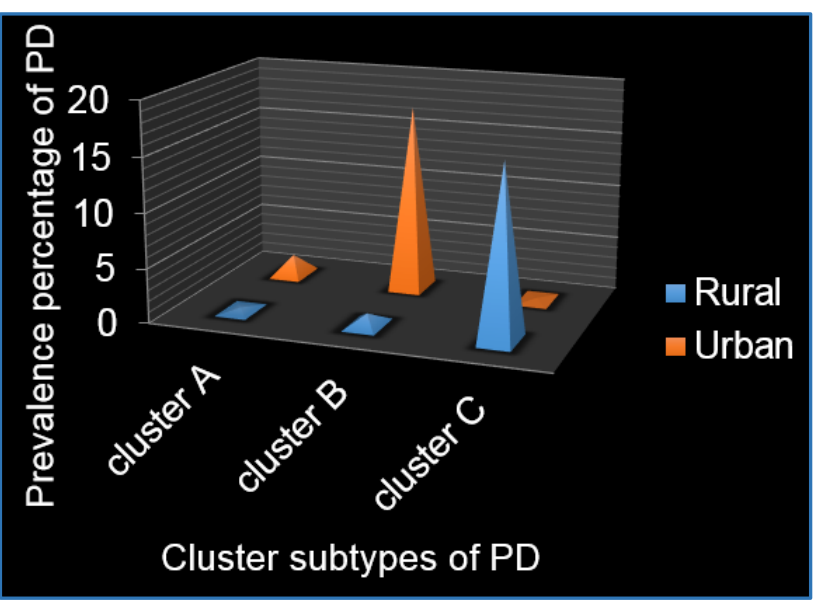

The overall prevalence rate of personality disorder in the community of Shimoga district was found to be $18.3 \%$ in the present study, where cluster C $(15.9 \%)$ is found to be the most prevalent in rural areas and cluster B $(17.3 \%)$ is found to be highly prevalent in urban areas where the cluster subgroups were consistent to the above-mentioned studies. These differences in cluster subgroups in the present study can be explained by the high number of illiterates, feel lack of powers, disfranchised and lack of independence in village areas, which results in cluster $\mathrm{C}$ personality traits.

Whereas cluster B was found to be very negligible in rural areas and can be due to plenty of support from the communities, having the joint families, where there is lots of mutual support to each other. Cluster B disorders were found to be highly prevalent in urban areas due to increased disorganised families, nuclear and broken families, children feel neglected by family members due to busy work schedules, more crimes and a poor community support makes people from urban areas more vulnerable to a cluster B subgroup of personality disorders.

Personality disorders are found to be frequent in a clinical population, especially in psychiatric outpatients where the prevalence rates were ranged from $15 \%$ to $45.5 \%$. Most of the previous studies have demonstrated that a substantial number of population in the community has personality disorders (Torgersen et al, 2001), which were lower than that found in clinical populations, but those studies were done only in urban settings. ${ }^{13}$

Previous research has suggested that younger age, lower socioeconomic status, male gender and being single amongst others are all predictors of PD. In our sample female (69.2\%) gender was predictive of a PD in rural areas, especially cluster C PD; whereas cluster B found to be predominating in males $(30.8 \%)$ among the urban population. A study by Samuels et al has found more males to have both cluster A and B PD and Coid et al found all PD to be more prevalent in males where studies were conducted only in urban settings. Another study by Torgersen et al 2001 has found that cluster A and B was found to be predominantly in males, whereas cluster $C$ found to be predominant in females which has also been reported in earlier studies (Zimmerman M, Coryell NH; 1989-1991). But unfortunately, we failed to collect other significant sociodemographic correlates of PD which was noteworthy. ${ }^{14}$ 
Prevalence of Personality Disorders from Various Studies Estimates of the prevalence of individual PD in previous community studies have not been altogether consistent.

A community study by Coid J, et al, in a Great Britain had found that the prevalence of personality disorder (4.4\%) in a community was lower compared to our study (18.3\%) and also in previous surveys which were conducted in other countries. These rates range from $3.9 \%$ to $22.3 \%$ (Zimmerman and Coryell, 1989; Maier et al, 1992; Black et al, 1993; Moldin et al, 1994; Klein et al, 1995; Lenzenweger et al, 1997; Torgersen et al, 2001; Samuels et al, 2002). These differences between the prevalence rates in different studies as explained by them was due to differences in sampling procedures, diagnostic instruments and number of disorder categories included rather than true differences between populations. $1,2,6,13$

Another community study by Suliman S (2007) had found that cluster A was most prevalent (3.4\%) followed by cluster $\mathrm{C}(2.5 \%)$ and cluster B was the least prevalent (1.5\%), which was interesting in view of the finding that cluster A PD are the least frequently seen in clinical populations (owing to lower treatment-seeking behaviour in this subtype) and cluster B PD was more frequently seen in clinical populations. Their study findings were inconsistent to our study and other previous studies (Lenzenweger et al, Coid et al 2008) where they have found cluster $C$ to be most prevalent followed by clusters A and B. Samuels et al found cluster B to be most prevalent followed by clusters $\mathrm{C}$ and $\mathrm{A}$, which is consistent with our study findings. $1,2,6$

An Indian study by Chandrasekara et al (1998) was done on the prevalence of personality disorder on suicide attempters and was assessed on 341 survivors (93\% of all survivors, over a one-year period) after their first suicide attempt from a general hospital. Only $7 \%$ received a diagnosis of personality disorder according to ICD 10 IPDE. The inclusion of the first attempt cases may have led to a low rate of diagnosis of emotionally unstable personality disorder (and consequently of any personality disorder). However, the rates were higher in special populations such as university students (19.1\%); criminals (7.3-33.3\%); patients with substance use disorders (20-55\%) in their study. ${ }^{15}$

The five major studies have examined the prevalence and type of personality disorders in the community sample of United States. According to the majority of those studies, the overall prevalence of Axis II disorders in the general population is consistently around 10 percent. [Iowa Study (1989) - 11.1\%, LSPD study (1997) - 11\%, Baltimore Study* (2002) - 10\%, NCS - R (2007) - 9.1\% and NESARC study (2008/09) - total prevalence of PD not known]. In contrast to studies in the United States, community prevalence rates of personality disorders in other countries show moderately wide variation from $6.1 \%-13.4 \%$, yet the averaging of these two low/high percentage results in $9.7 \% .^{16}$

In the cross-national study sponsored by the World Health Organisation, Researchers examined the prevalence of personality disorders in community samples from 13 countries (e.g. Colombia, Lebanon, Mexico, Nigeria, China, South Africa, the United States, Belgium, France, Germany, Italy, the Netherlands and Spain) using International Personality Disorder Examination (IPDE), the prevalence rates ranged from $2.4 \%$ to $7.9 \%$, the average prevalence among the 13 countries was $6.1 \%$ with clusters $\mathrm{A}, \mathrm{B}$ and $\mathrm{C}$ at
3.6 percent, 1.5 percent and 2.7 percent, respectively, which were inconsistent to our study findings with respect to urban areas. ${ }^{17}$

\section{Causes for Differences in Prevalence Rates among Rural and Urban Areas}

A number of factors which results in variation of prevalence rates between urban and rural area studies.

First of all, definitions of urban and rural may vary. The United Nations has defined an urban locality as having at least 20,000 people and a city as having at least 1,00,000 people. However, some countries define as urban using definitions from national statistical institutions or research may be defined as rural in another country. Secondly, the concrete manifestation of urban and rural phenomena varies widely around the world. The Netherlands, for instance, does not have any metropolis such as London or New York, and the Dutch countryside is much more populated than the countryside of Arkansas. Thirdly, there may be other cultural differences between studies and countries (Dohrenwend and Dohrenwend). ${ }^{18}$

To explain inner-city and urban-rural variations in prevalence rates, there are two main theoretical concepts, which originated from the early ecological research of schizophrenia and from the Chicago School of Sociology: the drift hypothesis and the breeder hypothesis. The 'drift hypothesis' assumes on the one hand that sick and vulnerable people are more or less doomed to remain in socially unstable, deprived neighbourhoods, while better off people move away (Social residue theory). On the other hand, socially deprived neighbourhoods can also have a pullfunction on sick and vulnerable people, as they move to these areas with low social control and greater tolerance towards deviant behaviour (Social drift hypothesis). Evidence concerning drift processes is still sparse. It remains to be seen; however, if these supposed drift processes apply to all psychiatric illnesses. Another theory, 'the breeder hypothesis' assumes that various environmental factors cause illness. These can be physical factors (Air pollution, small housing and population density) and also social factors (Stress, life events, perinatal aspects, social isolation). ${ }^{19}$

\section{Mindsets}

Urban Models and Mindset: There is a general belief that urban settings have much greater need for mental health services than rural areas and deserve more funding. Rural mental health problems and associated social problems (e.g. poverty, homelessness, domestic violence, drug abuse) tend to be less visible (they are not things you encounter on the street everyday or see on the local news every night) and can lead to the erroneous conclusion that rural areas have a lesser need for mental health services. There is also the belief based upon economic rationality, that it is simply not cost-effective to develop mental health services for rural populations and that the money it costs would be better spent serving a larger number of people in an urban setting.

Rural Mindsets: Rural areas are relatively free of the turf battles and guild issues that have so fragmented the general field of mental health. The rural community itself is an important strength of rural mental health. Rural communities by and large know their members and support each other. Community members and institutions (e.g. churches) often 
step into support consumers and their families in times of crisis and illness. The lack of anonymity works in positive ways to ensure that consumers do not become isolated and that clients having problems are quickly brought to the attention of rural mental health practitioners. ${ }^{20}$

Persons with certain types of serious mental illness find in rural settings an escape from the detrimental aspects of over stimulation, frenetic activity and fast pace of life that dominates metropolitan living. In addition, rural settings generally have less crime. ${ }^{21}$

\section{Causes as per International Prevalence Studies}

The most common personality disorder in a given culture, oftentimes differ from other cultures. For example, while an obsessive-compulsive personality disorder is the most common Axis II disorder in the United States and Australia, avoidant personality disorder is most common in Norway and schizotypal personality disorder is most common in Iceland. ${ }^{16}$

According to Jackson and Jovev, in war-torn countries, antisocial personality may allow for a distinct survival advantage, ultimately resulting in a population offset. Finally, Jackson and Jovev propose that particular cultures may attract individuals with specific personality disorders. ${ }^{22}$

\section{LIMITATIONS}

Several limitations of the present study must be considered. We were unable to interview subjects in the target sample who were deceased, unable or unwilling to participate or could not be traced.

The sample interviewed was restricted to rural and urban health centres rather than surveying at residential areas and did not include people in psychiatric institutions, the homeless or those in prison and also there was the possibility that individuals with an axis I or II disorder might have been more likely to decline participation in this study, resulting in an underestimation of prevalence rates.

Some subjects may have under or over reported specific personality features either deliberately or because they lacked insight. In addition, assessment of personality features of subjects and their informants might have been influenced by current symptoms.

It has been argued that collateral information from family members should be included when making diagnoses of these conditions. However, Zimmerman (1994) concluded that agreement between the two sources of information is generally poor and that the data remain insufficient to recommend one over the other.

\section{Implications}

Despite these limitations, the results provide estimates of the occurrences of DSM-V personality disorders in this community sample. Overall, we estimate that $18.3 \%$ of the subjects have maladaptive patterns of personality traits that were severe enough to cause them and others around them difficulties.

Management options will depend on a large number of factors such as the availability of health care resources, the therapist's own skill and stance and aspects of the patient's personality and present situation. These include his/her social support, associated psychiatric or physical illness, psychological mind-set, past relationship patterns and areas of resourcefulness. Pradhan et al presented a one-year follow- up of six male borderline patients treated with pharmacotherapy and psychotherapy, who improved substantially. More literature on individual management and service provision for personality disorders is urgently needed to counter the prevalent nihilism with regard to treatment outcome.

Western studies suggest that many patients maintain sustained improvement with treatment.

\section{CONCLUSIONS}

The current study indicated that specific subgroups of personality disorders in the community are over represented. These subgroups would be useful for investigating the determinants and consequences of these disorders and the service needs of people suffering from them as services are normally restricted to symptomatic, help-seeking individuals. The field of personality disorders is at a nascent stage of development in India. However, to date the focus is understandably but entirely on clinical epidemiology. There is obviously a need for better and more studies in relation to personality disorders on methodology and epidemiology (particularly community studies) and also on cultural issues. There is also a need for studies to populate the vast open swathes in terms of aetiology; the boundary issues between personality disorders and normal personality traits on the one hand and mental state disorders on the other; and the organisation of personality disorder in dimensional or categorical terms. ${ }^{23,24}$

The most common personality disorder varies from country to country and there may be a number of plausible explanations to explain this variation. Overall, having a sense of the community prevalence of Axis II disorders may help temper clinicians' expectations regarding the various personality types likely to be encountered in their respective practices, either psychiatric or primary care. 25,26

\section{ACKNOWLEDGEMENT}

I would like to say special thanks to the nursing staff from all the health centres who has supported for the sample collections. The author is grateful to the reviewers for their constant support and guidance.

\section{REFERENCES}

1. Coid J, Yang M, Tyrer P, et al. Prevalence and correlates of personality disorder in great britain. British Journal of Psychiatry 2006;188:423-31.

2. Suliman S, Stein DJ, Williams DR, et al. DSM-V personality disorders and their axis i correlates in the South African population. Psychopathology 2008;41(6):356-64.

3. Samuels, Eaton WW, Bienvenu III OJ, et al. Prevalence and correlates of personality disorders in a community sample. British journal of psychiatry 2002;180:536-42.

4. Peen J, Schoevers RA, Beekman AT, et al. The current status of urban-rural differences in psychiatric disorders. Acta Psychiatr Scand 2010;121(2):84-93.

5. Rahman A, Atram A. Prevalence of psychiatric disorders in a sample of elderly residents in rural and urban population of zulfi region Saudi Arabia. J Psychol Psychother 2014;5:1.

6. Lenzenweger MF, Lane MC, Loranger AW, et al. DSM-IV personality disorders in the national comorbidity survey replicatio. Biol Psychiatry 2007;62(6):553-64. 
7. Hesse M, Moran P. Screening for personality disorder with the standardised assessment of personality: abbreviated scale (SAPAS): further evidence of concurrent validity. BMC Psychiatry 2010;10:10.

8. Moran P, Leese M, Lee T, et al. Standardised assessment of personality abbreviated scale (SAPAS): preliminary validation of a brief screen for personality disorder. British Journal of Psychiatry 2003;183:228-32.

9. Merlhiot G, Mondillon L, Vermeulen N, et al. Adaptation and validation of the standardized assessment of personality-abbreviated scale as a self-administered screening test (SA-SAPAS). J Psychol Psychother 2014;4:6.

10. Jeste DV, Lieberman JA, Fassler D, et al. Diagnostic and statistical manual of mental disorders. American psychiatric association. $5^{\text {th }}$ edn. DSM-5TM, United states 2013:300, 645-84.

11. Rossier J, Ouedraogo A, Dahourou D, et al. Personality and personality disorders in urban and rural Africa: results from a field trial in burkina faso. Frontiers in psychology 2013;4:79.

12. Paris J. Personality disorders in sociocultural perspective. J Pers Disord 1998;12(4):289-301.

13. Torgersen S, Kringlen E, Cramer V. The prevalence of personality disorders in a community sample. Arch Gen Psychiatry 2001;58(6):590-6.

14. Zimmerman $\mathrm{M}$, Pfohl $\mathrm{B}$, Coryell $\mathrm{W}$, et al. Diagnosing personality disorders in depressed patients: a comparison of patients and informant interviews. Arch Gen Psychiatry 1989;45(8):733-7.

15. Chandrashekar CR, Reddy MV. Prevalence of personality disorders in suicide attempter. Indian J Psychiat 1998;40(Suppl 2):149-15.

16. Sansone RA, Sansone LA. Personality disorders: a nationbased perspective on prevalence. Innov Clin Neurosci 2011;8(4):13-8.
17. Huang Y, Kotv R, Kessler RC. DSM-IV personality disorders in the WHO World Mental Health Surveys. Br J Psychiatry 2009;195(1):46-53.

18. Maggi S, Ostry A, Callaghan $\mathrm{K}$, et al. Rural-urban migration patterns and mental health diagnoses of adolescents and young adults in British Columbia, Canada: a case-control study. Child and Adolescent Psychiatry and Mental Health 2010;4:13.

19. Savita, Duhan K. Personality assessment of rural and urban adolescent boys fromdisorganized families. J Sociology Soc Anth 2012;3(suppl1):43-7.

20. Vega WA, Kolody B, Aguilar-Gaxiola S, et al. Lifetime prevalence of DSM-III-R psychiatric disorders among urban and rural Mexican Americans in California. Arch Gen Psychiatry 1998;55(9):771-8.

21. Gamm L, Stone S, Pittman S. Mental health and mental disorders-a rural challenge: a literature review. Rural Healthy People 2010;96-114.

22. Beeson $\mathrm{PG}$, Britain $\mathrm{C}$, Howell ML, et al. Understanding rural mental health in the United States. Mental Health United States 1998;82-97.

23. Zimmerman M, Louis RL, Chelminski I. The prevalence of DSM-IV personality disorders in psychiatric outpatients. Am J Psychiatry 2005;162(10):1911-8.

24. McGilloway A, Ruth EH, Lee T, et al. Asystematic review of personality disorder, race and ethnicity: prevalence, aetiology and treatment. BMC Psychiatry 2010;10:33.

25. Tyrer P, Reed GM, Crawford MJ. Personality disorder: classification, assessment, prevalence, and effect of personality disorder. Lancet 2015;385(9969):717-26.

26. Park S, Hong JP, Lee HB, et al. Relationship between personality disorder dimensions and verbal memory functioning in a community population. Psychiatry Res 2012;196(suppl1):109-14. 